\title{
Über-Reden durch An-Reden \\ Die phatische Funktion als Mittel zum Appell in englischen, spanischen und deutschen Werbetexten
}

\author{
Christiane Nord \\ Hochschule Magdeburg-Stendal, Magdeburg, Deutschland
}

Advertisements are intended to persuade the addressees to buy a particular product or service. In order to achieve this communicative purpose, text-producers make use of various strategies. Apart from directly appellative speech acts like recommendations, requests or invitations, they use referential, expressive, and phatic utterances as an indirect means of persuasion. The phatic function, which, according to Jakobson (1960), provides the appropriate channel for communication, is of particular importance in this respect. If the channel does not work properly, the persuasive function will never reach its aim. In the following paper, a small corpus of English, Spanish and German advertising texts is analyzed in order to find the indicators of the phatic function which are instrumental in making persuasion work.

\section{Allgemeines}

Es ist eine allgemein bekannte Tatsache, dass Werbetexte dazu dienen sollen, eine Zielperson dazu zu bringen, ein Produkt zu erwerben oder eine Dienstleistung in Anspruch zu nehmen, auch wenn sie vorher vielleicht nicht einmal wusste, dass es ein solches Produkt gibt, geschweige denn das Bedürfnis hatte, es zu erwerben. Also, so denkt sich der Laie, muss man sie erst einmal darüber informieren, dass das Produkt existiert. Der neue Renault Espace ist $d a$, heißt es unter der Abbildung eines schicken Autos auf einem Plakat, und wir erinnern uns daran, dass auch der neue Beaujolais so angekündigt wird: Le Beaujolais nouveau est arrivé, sogar in Deutschland. Es ist eigentlich überraschend, dass das neue Produkt mit dem bestimmten Artikel eingeführt wird, so als wüssten wir bereits darüber Bescheid, als hätte uns bereits vorher jemand angekündigt: "Bald kommt ein neues Modell des Renault Espace auf den Markt." Und nun ist er da, der neue Renault Espace. Gibt es da vielleicht eine kommunikative Verbindung zwischen der Firma und uns, von der wir bisher nichts geahnt haben?

Noch ein Beispiel: In einer spanischen Werbeanzeige einer Autovermietung heißt es: Si pensabas que nunca podrías conducir un Peugeot 406 Coupé te equivocabas. Woher wissen die, was ich denke? Und warum reden sie so vertraulich mit mir, als würden sie mich schon ewig kennen?

Wenn wir einmal darauf achten, stellen wir fest, dass dieses Phänomen in vielen Werbetexten zu beobachten ist. Die Texte klingen, als wären sie Teil einer bereits andauernden Kommunikation zwischen Menschen, die sich schon länger kennen. Auf Deutsch wird der Adressat in 
Werbetexten meist mit der Höflichkeitsform Sie angeredet, aber dennoch ist der Ton vertraulich: Sie möchten Ihre Jobsuche ganz entspannt angehen? Damit können Sie jetzt sofort loslegen: einfach Ihr Gesuch auf unserer Homepage ablegen... Die Wörter Job und loslegen sind umgangssprachlich markiert (vgl. Duden 1993), die Modalpartikel einfach kennzeichnet einen ungezwungenen, sprechsprachlichen Stil - so redet man nicht mit Leuten, die man nicht kennt.

Der Kontakt zwischen Sender und Empfänger in einer Kommunikationssituation ist das Feld der so genannten phatischen Funktion. Nach Jakobson (1960) ist die phatische Funktion für den Kommunikationskanal zuständig und sorgt dafür, dass ein kommunikativer Kontakt hergestellt, aufrecht erhalten und gegebenenfalls beendet wird. Aus meiner Sicht gehört zu diesem Aufgabenfeld auch die Definition und Ausgestaltung der sozialen Beziehung zwischen Sender und Empfänger, also genau der Bereich, in dem festgelegt wird, wie man jemanden anredet: welches Pronomen angemessen ist, ob man Titel erwähnt oder welches stilistische Register angemessen ist.

Im Folgenden wollen wir zunächst der Frage nachgehen, wie die phatische Funktion mit der persuasiven Funktion von Werbetexten zusammenhängt. In einem zweiten Schritt werden wir untersuchen, wie die einzelnen Unterfunktionen der phatischen Funktion, also Kontakteröffnung, Kontaktbeendigung, Kontakterhalt und Beziehungsgestaltung, in Werbetexten sprachlich realisiert werden und ob bzw. wie sich die sprachliche Realisierung in verschiedenen Kulturen unterscheidet.

Wir gehen dabei davon aus, dass bei der Wahl bestimmter Realisierungsformen Konventionen im Spiel sind, die von den durch die Sprachsysteme zur Verfügung gestellten Strukturen unabhängig, durch historische "Verhaltensgewohnheiten" bedingt (vgl. Lewis 1969) und daher kultur- und nicht sprachspezifisch sind. Auch wenn nur eine sprachliche Form zur Verfügung steht (wie etwa im Englischen beim Anredepronomen you), hat ein Sprecher immer noch die Möglichkeit, sie zu verwenden oder zu umgehen, also ist auch hier eine Entscheidung zu treffen, die durch kulturelle Konventionen geprägt sein dürfte.

Konventionen lernt man nur durch Beobachtung - für die Analyse der Konventionen phatischer Kommunikation braucht man also ein Korpus authentischer Kommunikationsakte. Wir haben nach dem Zufallsprinzip aus aktuellen Zeitungen und Zeitschriften ein kleines Korpus von je 100 Werbetexten für 22 verschiedene Produkt- und Dienstleistungskategorien ${ }^{1}$ aus den Jahren 1996 bis 2001 ausgewählt, die sich an Zielgruppen in Großbritannien, Spanien und Deutschland richten (vgl. Bibliographie).

Die Spezifizierung nach Ländern erscheint deshalb gerechtfertigt, weil Werbetexte ein gutes Beispiel für adressatenorientierte Textgestaltung sind. Gerade bei großen Sprachräumen, die sich über mehrere Länder und sogar Kontinente erstrecken, können wir nicht von einer Kongruenz der Sprach- und Kulturräume ausgehen. Es ist auch eine Erfahrungstatsache, dass für das selbe Produkt einer Firma in verschiedenen Ländern unterschiedlich geworben wird. Eine Ausnahme bilden "Hochpreisprodukte" wie 
teure Parfums oder Juwelen, bei denen weltweite Werbekampagnen zu beobachten sind, in denen sich die Werbeanzeigen kaum oder gar nicht unterscheiden. Die "Schönen und Reichen" bilden offensichtlich eine eigene (Dia)Kultur, die quer zu Sprach- oder Ländergrenzen gelagert ist.

Die Untersuchung ist in den Rahmen einer komparativen Stilistik einzuordnen (vgl. Nord 2003, im Druck). Allerdings unterscheidet sie sich im Ansatz grundsätzlich von der Stylistique Comparée von Vinay \& Darbelnet (1958), die neuerdings wieder fröhliche Urständ zu feiern scheint (vgl. zum Beispiel Álvarez Lugrís 2001). Hier geht es nicht um eine Méthode de traduction, sondern um etwas, das vor jeder Übersetzung liegt, für diese jedoch unerlässlich ist. Anders als die Kontrastiven Grammatiken (vgl. z.B. Cartagena \& Gauger 1989) vergleicht die komparative Stilistik Einheiten der Parole und nicht der Langue und stützt sich nicht auf die Kontrastierung von Originalen und ihren Übersetzungen, sondern auf den Vergleich authentischer Paralleltexte. Als Paralleltexte betrachten wir Texte aus zwei Kulturen, die für die gleichen oder vergleichbare kommunikative Funktionen gedacht sind und daher der gleichen oder einer zumindest vergleichbaren Textsorte angehören. Dabei geht es uns jedoch nicht um Textsortenkonventionen, sondern um die Konventionen der Versprachlichung bestimmter kommunikativer Funktionen bzw. Unterfunktionen, die in allen oder vielen Textsorten zu finden sind.

Unter Stil verstehen wir die Auswahl aus zwei oder mehreren grammatikalisch korrekten Ausdrucksmöglichkeiten für eine bestimmte Kommunikationsintention. Immer dann, wenn es mehr als eine Möglichkeit gibt, eine bestimmte kommunikative Intention $\mathrm{zu}$ versprachlichen, wird eine stilistische Entscheidung getroffen, die sich - unter anderem - auch an den in der betreffenden Kultur vorherrschenden Konventionen orientiert.

\section{Phatische Funktion und Werbung}

Werbetexte werden im Allgemeinen der persuasiven Funktion zugeordnet, die ihrerseits zur Appellfunktion gehört. Werbung soll "überreden", also "jemanden durch [eindringliches Zu]reden dazu bringen, dass er etwas tut, was er ursprünglich nicht wollte" (Duden 1993). Um die Adressaten eines Werbetexts zum Kauf eines bestimmten Produkts zu überreden, muss man ihnen eindringlich zureden, sie bei ihren geheimen Wünschen und Wertvorstellungen packen, und das setzt voraus, dass man diese kennt. Das "eindringliche Zureden" darf aber nicht so plump wirken, dass die Adressaten in Abwehrhaltung gehen, sondern es muss dafür gesorgt werden, dass die Empfänger nicht merken, wie sie überredet werden. Von "geheimen Verführern" (hidden persuaders) sprach Vance Packard (1967) schon vor Jahrzehnten.

Aus diesem Grunde sind in Werbetexten offene Aufforderungen nach dem Muster "Kaufen Sie das Produkt XY" eher selten. So beträgt das quantitative Verhältnis zwischen den verschiedenen Formen des (indirekten) 
Überredens und denen des Aufforderns, zu dem wir etwa Empfehlungen, Ratschläge, Einladungen und dergleichen rechnen, in unserem englischen Korpus 5:1, im spanischen Korpus 3:1 und im deutschen Korpus 4:1 (vgl. Nord 2003, im Druck). Statt dessen werden andere kommunikative Funktionen, also die Darstellungsfunktion, die Ausdrucksfunktion und die phatische Funktion, wenn wir von unserem übersetzungsorientierten 4-Funktionen-Modell ausgehen (vgl. Nord 1997), als indirekte Mittel zur Appellrealisierung eingesetzt.

Wenn sich die phatische Funktion, wie schon angemerkt, nach Jakobson (1960) auf die Leistungsfähigkeit des Kanals bezieht, liegt ihre Bedeutung für die persuasive Kommunikation auf der Hand. Wo kein Kanal ist, kann auch keine Kommunikation stattfinden, und wenn bei der Herstellung des kommunikativen Kontakts mit der Zielgruppe "etwas schief läuft", kann eine persuasive Absicht gar nicht erst an ihr Ziel gelangen. Daher scheint die phatische Funktion auch in persuasiven Texten eine Schlüsselrolle zu spielen.

Phatische Äußerungen sind Begrüßungen und Verabschiedungen, Formeln, mit denen man sich am Telefon meldet oder ein Gespräch beendet oder jemandem seine Frau vorstellt oder von einem Thema zum anderen überleitet. Eine typische phatische Kommunikation ist der so genannte Smalltalk über das Wetter zwischen Menschen, die sich nicht kennen, aber vielleicht zusammen im Zugabteil sitzen oder auf einem Empfang mit einem Glas in der Hand neben einander stehen. Aus dem Smalltalk kann eine "richtige" Unterhaltung werden oder auch nicht.

Die wenigen Beispiele zeigen bereits, dass das "Funktionieren" der phatischen Kommunikation wesentlich von der Konventionalität der verwendeten sprachlichen und nichtsprachlichen Verhaltensweisen abhängt. Man erkennt das besonders gut daran, wenn jemand sich nicht konventionell verhält, wenn er also auf die Grußfrage $\mathrm{Na}$, wie geht's? mit einer detaillierten Schilderung seines Gesundheitszustands antwortet, oder den SmalltalkAuftakt Schönes Wetter heute, nicht? mit einem Das finde ich überhaupt nicht! kontert und nicht, wie es üblich ist, zustimmend reagiert, auch wenn er das Wetter in Wirklichkeit tatsächlich nicht besonders schön findet.

Die Tatsache, dass in Übersetzungen und Filmsynchronisationen oft solche Negativbeispiele zu hören oder zu lesen sind (z.B. Haben Sie meinen Mann schon getroffen? als Vorstellung, statt Darf ich Ihnen meinen Mann vorstellen? oder Kennen Sie eigentlich meinen Mann schon?), spricht dafür, dass wir diesen Bereich der Kommunikation in der Übersetzerausbildung offensichtlich zu sehr vernachlässigen. Dabei ist der Aufbau und die Ausgestaltung der sozialen Beziehung zum Gesprächspartner ein Aspekt der Kommunikation, der meist nicht einmal in der eigenen Kultur "bewusst" vonstatten geht, der aber den Eindruck, den der Adressat vom Sender bekommt, maßgeblich mit bestimmt.

Im Bereich der sozialen Beziehungen ist zwischen Status und Rolle zu unterscheiden. Der Status ist einer Person auf Grund von Bildung, Beruf, Stellung etc. zu einem bestimmten Zeitpunkt fest zugeschrieben, während sich die Rolle von Situation zu Situation ändern kann. Ein Angestellter, der 
gegenüber seinem Chef in der Rolle des "Untergebenen" agiert, kann zu Hause am Mittagstisch gegenüber dem Sohn oder im Restaurant gegenüber dem Kellner als "Boss" auftreten - dabei hat sich an seinem Status nichts geändert.

Vom Status hängt zunächst einmal das phatische Verhalten zwischen zwei Personen ab, die einander vorgestellt werden - in der weiteren Kommunikation kann sich dann eine Rollenbeziehung entwickeln, die dem Status entspricht oder auch nicht. Dabei spielen die verwendeten Anredeformen eine wichtige Rolle, weil sie die soziale Beziehung nicht nur wiederspiegeln, sondern auch gestalten. Dabei können für den Übergang von einer asymmetrischen Rollenbeziehung (zwischen "oben" und "unten") zu einer symmetrischen Rollenbeziehung, bei der die Partner auf gleicher Ebene stehen, bestimmte Rituale oder Formeln üblich sein (z.B. Wir könnten eigentlich 'du' sagen, oder?).

In Kulturen, in denen nicht zwischen formellen und vertraulichen Anredeformen unterschieden wird, muss die soziale Beziehung durch andere Mittel markiert werden (z.B. durch Nennung von Titeln oder Anrede beim Vornamen oder, wie sich bei der Analyse des Werbetextkorpus zeigen wird, durch die Wahl des Registers).

Wir wollen daher das Korpus aus englischen, spanischen und deutschen Werbetexten auf folgende Aspekte hin untersuchen:

- Mit welchen sprachlichen Mitteln wird die Kommunikation zwischen Sender und Empfänger eröffnet, fortgeführt und beendet (Kontaktindikatoren)?

- Wie oft und in welcher Form werden die Adressaten der Werbetexte direkt angeredet (Adressierungsindikatoren)?

- Mit welchen sprachlichen und/oder stilistischen Mitteln wird die angestrebte Rollenbeziehung zwischen Sender und Empfänger markiert (Beziehungsindikatoren)?

Die wichtigsten Analyseparameter sind also Form und Frequenz. Sie bilden auch die Kriterien des Kulturvergleichs.

\section{Korpusanalyse}

Im Folgenden sollen die drei genannten phatischen Indikatoren in Bezug auf ihre Form und Frequenz in persuasiven Texten untersucht werden.

\subsection{Kontaktindikatoren in persuasiven Texten}

Wie bereits bemerkt, dienen die Kontaktindikatoren dazu, den kommunikativen Kanal zu öffnen, offen zu halten und zu schließen. Wir können daher kontakteröffnende, kontakterhaltende und kontaktbeendende Indikatoren unterscheiden. 


\subsubsection{Kontakteröffnung}

Der Kontakteröffnung dienen zum Beispiel Begrüßungen (en. ${ }^{2}$ Cheers!, de. Willkommen, 3.1-5), Zuwendungssignale, wie z.B. es. dígame, oiga, tranquilo (3.1-3) oder unartikulierte Laute, um die Aufmerksamkeit des Adressaten zu erregen (3.1-2), nominale Anredeformen, wie es. señor, caballero oder en. Sir, women, guys (3.1-1), de. Herr Meier, gnädige Frau, Personalpronomina im Vokativ, wie de. He, Sie da oder es. tú, dime, oder Dankesformeln (3.1-4).

\begin{tabular}{|l|l|}
\hline $3.1-1$ & $\begin{array}{l}\text { Women! Cheaper car insurence. Some men will try anything to } \\
\text { get cheaper car insurance! Sorry guys! You can try as hard as you } \\
\text { like, but Diamond is just for women. Diamond only do car insu- } \\
\text { rance for women because women are better drivers. (FIN) }\end{array}$ \\
\hline $3.1-2$ & $\begin{array}{l}\text { [Psst!] You are very close to experiencing something quite } \\
\text { exceptional. (ALK) }\end{array}$ \\
\hline $3.1-3$ & $\begin{array}{l}\text { Ésta es la distancia que te separa de tu oficina. Tranquilo. No } \\
\text { pasa nada porque te reserves un poco de tiempo para ti mismo. // } \\
\text { Acortamos distancias. Acercamos personas. (TEL) }\end{array}$ \\
\hline $3.1-4$ & $\begin{array}{l}\text { »En mi nombre y en el del Comité de dirección de SSA Ibérica, } \\
\text { quiero agradecer a todos nuestros clientes, afiliados y partners la } \\
\text { confianza depositada en nuestra solución... «(FIN) }\end{array}$ \\
\hline $3.1-5$ & \begin{tabular}{l} 
Willkommen bei ANA, der größten Fluglinie Japans. (TRA) \\
\hline
\end{tabular}
\end{tabular}

Auch rhetorische Fragen, die wie ein Auftakt zum Smalltalk wirken, sind phatische Kontakteröffnungssignale. Im Deutschen erkennt man sie an der fehlenden Inversion und daran, dass sie eine Bejahung nahe legen (z.B. 3.18). Im spanischen Korpus sind sie meist nicht direkt an den Adressaten gerichtet, suggerieren jedoch ebenfalls eine bestimmte Antwort, die allerdings auch verneinend sein kann (z.B. 3.1-9). Andere Fragen, die meist durch die Abbildung motiviert sind (z.B. 3.1-10 etwa durch den traurigen Gesichtsausdruck eines Mannes), führen in das Thema ein und benennen ein Problem des Adressaten (3.1-10, 3.1-11). Auch sie haben kontakteröffnende Funktion.

\begin{tabular}{|l|l|}
\hline $3.1-6$ & Don't you love just things that make life easier? (HAU) \\
\hline $3.1-7$ & $\begin{array}{l}\text { ¿Quiere probar cuánta cobertura tiene el inalámbrico Digital } \\
\text { Samsung? Lléveselo de paseo. (TEL) }\end{array}$ \\
\hline $3.1-8$ & $\begin{array}{l}\text { Sie möchten Ihre Jobsuche ganz entspannt angehen? Damit kön- } \\
\text { nen Sie jetzt sofort loslegen: einfach Ihr Gesuch auf unserer } \\
\text { Homepage ablegen. (KOM) }\end{array}$ \\
\hline
\end{tabular}




\begin{tabular}{|l|l|}
\hline $3.1-9$ & $\begin{array}{l}\text { ¿Hay una manera mejor de terminar el año? (AUT, mit Bezug } \\
\text { auf die Abbildung) }\end{array}$ \\
\hline $3.1-10$ & ¿Piel apagada, deshidratada? (KOS) \\
\hline $3.1-11$ & $\begin{array}{l}\text { Condensation and dampness? Solved with an EBAC dehumidi- } \\
\text { fier. (HAU) }\end{array}$ \\
\hline
\end{tabular}

Ähnlich kontakteröffnend wirkt der Hinweis auf eine Situation, in der das angebotene Produkt für den Adressaten die Lösung seines Problems bringt. Hier werden meistens konditionale Satzgefüge mit Wenn..., dann... verwendet. Diese Konditionalgefüge finden sich in allen drei Korpora (Bsp. 3.1-12 bis 3.1-14).

\begin{tabular}{|l|l|}
\hline $3.1-12$ & $\begin{array}{l}\text { If you' re thinking about borrowing money, we could help you. } \\
\text { (FIN) }\end{array}$ \\
\hline $3.1-13$ & $\begin{array}{l}\text { Si quiere saber por qué España está de moda, haga sus compras } \\
\text { en El Corte Inglés. (KOM) }\end{array}$ \\
\hline $3.1-14$ & $\begin{array}{l}\text { Wenn es auch bei Ihnen mal schneller gehen soll, dann kommen } \\
\text { Sie am besten zu unserem neuen Express-Service. (AUT) }\end{array}$ \\
\hline
\end{tabular}

Tabelle 1 gibt einen Überblick über die Frequenz der Kontakteröffnungsindikatoren in den drei Teilkorpora. Da jedes Teilkorpus genau 100 Texte umfasst, entsprechen die absoluten Zahlen auch dem prozentualen Anteil. Eine besondere Häufigkeit bestimmter Kontaktindikatoren in einer bestimmten Produktkategorie ist nicht festzustellen.

\begin{tabular}{|l|c|c|c|}
\hline Kontakteröffnungsindikatoren & Englisch & Spanisch & Deutsch \\
\hline Begrüßung & 1 & 0 & 1 \\
\hline Zuwendungssignale & 1 & 1 & 0 \\
\hline nominale Anrede & 1 & 0 & 0 \\
\hline pronominale Anrede & 1 & 0 & 0 \\
\hline Dank & 1 & 1 & 0 \\
\hline rhetorische Frage & 2 & 4 & 5 \\
\hline thematische Frage & 7 & 5 & 2 \\
\hline Bedingungsgefüge & 1 & 3 & 2 \\
\hline gesamt & 15 & 14 & 10 \\
\hline
\end{tabular}

Tabelle 1: Kontakteröffnungsindikatoren in Werbetexten 


\subsubsection{Kontakterhalt}

Es liegt auf der Hand, dass der Sender einer Werbeäußerung den Kontakt zum Adressaten gern möglichst lange aufrecht erhalten möchte, damit dieser sich daran erinnert, wenn er die Gelegenheit hat, das Produkt zu erwerben. Für diese Art von Kontakterhalt ist besonders der Slogan geeignet. Ein Slogan ist "eine einprägsame, wirkungsvoll formulierte Redewendung" (Duden 1993), der oft in allen oder mehreren Werbetexten eines Produktanbieters verwendet wird. Damit ein Slogan sich ins Gedächtnis einprägt, ist er durch rhetorische Mittel wie Rhythmus, Reim, Assonanzen, Alliterationen, Parallelismen, Wortspiele oder Anspielungen gekennzeichnet.

\begin{tabular}{|l|l|}
\hline $3.1-15$ & $\begin{array}{l}\text { With ice. With cola. With feeling. Vodka toffee. Not for the } \\
\text { Sweet \& Innocent. Dooley's toffee liqueur. (NAH) }\end{array}$ \\
\hline $3.1-16$ & $\begin{array}{l}\text { A rowing-boat for a liqueur. (Possibly the best swap in history.) } \\
\text { (ALK) }\end{array}$ \\
\hline $3.1-17$ & $\begin{array}{l}\text { FREEZE! Icy pink meets silver plum, glitter got the shivers. } \\
\text { (KOS) }\end{array}$ \\
\hline $3.1-18$ & $\begin{array}{l}\text { Get the glitter bug. Glitterations Christmas 2001. The season is } \\
\text { upon us, get ready to party. Glitz and glam, sexy and sultry. } \\
\text { Whatever look you want to create, keep yourself sparkling. Get } \\
\text { it on. Rimmel. (KOS) }\end{array}$ \\
\hline $3.1-19$ & $\begin{array}{l}\text { Conquer Rome. All roads lead to the new Mövenpick Hotel } \\
\text { Central Park in Rome... Guests may enjoy the "Dolce far niente" } \\
\text { on the terrace of the Gran Café... (TOU) }\end{array}$ \\
\hline $3.1-20$ & $\begin{array}{l}\text { Noble su reposo, de roble su carácter. Canarias Cuba del Ron. } \\
\text { (ALK) }\end{array}$ \\
\hline $3.1-21$ & $\begin{array}{l}\text { O tomas ron con Schweppes Limón o Schweppes Limón con } \\
\text { ron. (NAH) }\end{array}$ \\
\hline $3.1-22$ & $\begin{array}{l}\text { Culto al ego. VegaFina. Hecho a mano. Sabor suave, placer } \\
\text { intenso. (TAB) }\end{array}$ \\
\hline $3.1-23$ & $\begin{array}{l}\text { Haz "click" y huele. En el futuro se podrán percibir aromas a tra- } \\
\text { vés de los nuevos periféricos de los ordenadores. (FIN) }\end{array}$ \\
\hline Groß. Stark. Unverzichtbar! Bild wirkt. (INF) \\
\hline $\begin{array}{l}\text { Lieber trocken trinken als trocken feiern. (ALK) } \\
\text { Do it with German engineering. (deutsches Korpus, AUT) }\end{array}$ \\
\hline $3.1-26$
\end{tabular}

Nicht alle Texte des Korpus haben einen Slogan; in 17 spanischen, 20 englischen und 22 deutschen Texten ist kein Slogan zu erkennen. Oft ist auch nicht ganz klar, welche Formulierung eventuell ein Slogan ist, weil das 
Korpus ja nicht immer mehrere Werbetexte des gleichen Herstellers umfasst. Daher werden in Tabelle 2 die in den Texten verwendeten rhetorischen Mittel allgemein aufgeführt: rhythmische Muster (3.1-10, 3.1-17), syntaktische Figuren (Parallelismus: 3.1-20, Triade: 3.1-15, 3.1-24, Ellipse, Chiasmus, wie in 3.1-21), Klangfiguren (Reim: 3.1-21, Assonanz: 3.1-20, Alliteration: 3.1-25, Lautmalerei: 3.1-17, 3.1-18), lexikalische Figuren (Konnotationen, oft sexueller Art: 3.1-15, 3.1-22, 3.1-26, Wortspiele: 3.1-25), Intertextualität (Anspielung: 3.1-15, 3.1-16, Zitat: 3.2-23). Es fällt auf, dass in einigen spanischen und zahlreichen deutschen Werbetexten der Slogan in englischer Sprache formuliert ist (3.1-26) oder englischsprachige Elemente enthält (3.1-23). Im englischen Korpus findet sich nur ein Text mit ein paar Worten auf Italienisch und eine Dior-Werbung in französischer Sprache, bei der es sich jedoch um eine internationale Werbung handelt. Da oft mehrere stilistische Mittel in einem Werbetext vorkommen, sind die Prozentanteile auf der Grundlage der Gesamtzahl der verwendeten rhetorischen Figuren berechnet.

\begin{tabular}{|l|r|r|r|r|r|r|}
\hline Kontaktverlängerungsindikator & \multicolumn{2}{|c|}{ Englisch } & \multicolumn{2}{|c|}{ Spanisch } & \multicolumn{2}{|c|}{ Deutsch } \\
\hline rhythmische Muster & 6 & $10,0 \%$ & 5 & $14,3 \%$ & 2 & $3,6 \%$ \\
\hline syntaktische Stilfiguren & 14 & $23,3 \%$ & 9 & $25,7 \%$ & 10 & $18,2 \%$ \\
\hline Assonanz, Alliteration & 6 & $10,0 \%$ & 4 & $11,4 \%$ & 1 & $1,8 \%$ \\
\hline Reim & 5 & $8,3 \%$ & 1 & $2,9 \%$ & 0 & $0,0 \%$ \\
\hline Konnotationen & 4 & $6,7 \%$ & 8 & $22,9 \%$ & 5 & $9,1 \%$ \\
\hline Wortspiele & 17 & $28,3 \%$ & 2 & $5,7 \%$ & 15 & $27,3 \%$ \\
\hline Intertextualität & 6 & $10,0 \%$ & 2 & $5,7 \%$ & 14 & $25,5 \%$ \\
\hline fremdsprachliche Elemente & 2 & $3,3 \%$ & 4 & $11,4 \%$ & 8 & $14,5 \%$ \\
\hline gesamt & 60 & $100 \%$ & 35 & $100 \%$ & 55 & $100 \%$ \\
\hline
\end{tabular}

Tabelle 2: Kontaktverlängerungssignale in Werbetexten

Tabelle 2 zeigt, dass syntaktische Stilfiguren in allen drei Teilkorpora recht häufig sind, während Wortspiele in englischen und deutschen Werbetexten häufiger vorkommen als in spanischen. Klangfiguren sind in deutschen Werbetexten so gut wie gar nicht belegt, in spanischen häufiger und besonders häufig in den englischen Texten. Auffällig ist die hohe Frequenz von Wörtern mit emotionalen oder sexuellen Konnotationen im spanischen Korpus. Der hohe Anteil von Anspielungen im deutschen Korpus rührt daher, dass einerseits eine Reihe von intertextuellen Bezügen auf andere Slogans vorkommen und andererseits eine bestimmte Form des Slogans häufig ist, die an die Form eines Sprichworts erinnert: z.B. Wer damit zahlt, 
kriegt Meilen (nämlich mit der Lufthansa Visa Card), vergleichbar mit "Wer langsam geht, kommt auch zum Ziel" oder "Wer andern eine Grube gräbt, fällt selbst hinein".

\subsubsection{Kontaktbeendigung}

Echte Kontaktbeendigungssignale kommen interessanterweise gar nicht vor. Es gibt im deutschen Korpus zwar eine Abschiedsformel (3.1-27), aber hier handelt es sich eher um ein Wortspiel: die Programmzeitschrift wünscht den Lesern einen schönen "Fernsehabend".

\begin{tabular}{|l|l|}
\hline $3.1-27$ & TV Today. Schönen Abend noch... (INF) \\
\hline $3.1-28$ & Available at: Barrats or a stockist near you. (MOD) \\
\hline $3.1-29$ & $\begin{array}{l}\text { Servicio de atención al cliente } 902119580 . \\
\text { Internet: http://www.and.es (AUT) }\end{array}$ \\
\hline $3.1-30$ & $\begin{array}{l}\text { Weitere Informationen erhalten Sie kostenlos unter freecall } \\
080033 \text { 07001, im T-Punkt oder unter www.t-online.de (TEL) }\end{array}$ \\
\hline
\end{tabular}

Man könnte sogar sagen, dass die fast schon standardmäßigen Hinweise auf eine Homepage oder eine Telefonnummer, unter der man weitere Informationen bekommen kann (3.1-29, 3.1-30), als eine Art "Bis bald" wirken und damit auch eher kontaktverlängernde als kontaktbeendende Funktion haben.

\subsection{Adressierungsindikatoren in Werbetexten}

Ein Werbetext simuliert gewissermaßen eine erste Begegnung zwischen Menschen, die sich nicht kennen ${ }^{3}$. Im "richtigen Leben" wäre es üblich, dass sich die eine Person der anderen vorstellt (Selbstidentifikation) oder dass die beiden einander von einem Dritten vorgestellt werden (Vorstellung). Das kann man auch in einigen der Werbetexte finden. Die Herstellerfirma, die ein Produkt oder eine Dienstleistung anbietet, wird in der Regel durch den Firmennamen und/oder das Logo identifiziert, gelegentlich stellt sie sich aber auch explizit vor (z.B. "Wir sind ein Unternehmen, das...", vgl. auch 3.2-1), wobei dann meist die Firmenphilosophie erläutert (3.2-3) oder auf die besondere Kompetenz der Firma hingewiesen wird (3.2-2). Diese Form der Vorstellung ist allerdings in allen drei Korpora nicht besonders häufig belegt.

\begin{tabular}{|l|l|}
\hline $3.2-1$ & WHSmith. We make a big deal of books. (INF) \\
\hline $3.2-2$ & Lo único que hacemos es invertir. (FIN) \\
\hline $3.2-3$ & $\begin{array}{l}\text { Meine Uhren gehen alle hundert Jahre nach. (MOD, mit einer } \\
\text { Abbildung des Firmengründers) }\end{array}$ \\
\hline
\end{tabular}


Öfter vertreten ist die Vorstellung durch eine im Text nicht identifizierte Person, gewissermaßen "aus dem Off” (3.2-4, 3.2-5, 3.2-6), wobei hier die Vorstellung allerdings meist nicht so sehr informativ, sondern vielmehr anpreisend, also expressiv, ist. In einem Text stellt sich sogar das Produkt selbst vor (3.2-7).

\begin{tabular}{|l|l|}
\hline $3.2-4$ & Canon dice la última palabra en fotocopiadoras color. (BÜR) \\
\hline $3.2-5$ & $\begin{array}{l}\text { Profitieren Sie vom Know-how einer der erfahrensten Invest- } \\
\text { mentfonds-Gesellschaften der Welt (FIN) }\end{array}$ \\
\hline $3.2-6$ & $\begin{array}{l}\text { AZCO NOBEL knows the importance of stimulating young } \\
\text { talent. (BIL) }\end{array}$ \\
\hline $3.2-7$ & Spann mich ein, ich bin ein Transit. (AUT) \\
\hline
\end{tabular}

In einigen Texten spricht eine in einer Abbildung dargestellte Person, die als typischer Nutzer des Produkts betrachtet werden kann. Sie drückt ihre Zufriedenheit oder sogar Begeisterung über das Produkt aus. Dabei handelt es sich jedoch nicht um eine Identifikation im engeren Sinne, sondern eher um eine Bewertung, die wir der expressiven Funktion zuordnen würden.

\begin{tabular}{|l|l|}
\hline $3.2-8$ & "El mejor vendedor de $m i$ tienda es un auténtico plasma." (BÜR) \\
\hline $3.2-9$ & We borrowed $£ 20,000$ for just $£ 184$ a month. (FIN) \\
\hline $3.2-10$ & Ich mag den Golf Joker. (AUT) \\
\hline $3.2-11$ & Dior - j'adore the absolute femininity. (KOS) \\
\hline $3.2-12$ & $\begin{array}{l}\text { Thank you, Ardbeg, } I \text { have always tried to find a single malt with } \\
\text { a full bodied taste. At last } I \text { 've found it... (ALK) }\end{array}$ \\
\hline
\end{tabular}

In der Regel sind die Senderidentifikationen an den Adressaten des Texts gerichtet, nur in zwei Werbeanzeigen des englischen Korpus richtet sich ein typischer Kunde an die Firma (3.2-11, 3.2-12). Diese beiden Beispiele werden bei den Identifikationen nicht mitgezählt.

\begin{tabular}{|l|c|c|c|}
\hline Identifikation des Senders & Englisch & Spanisch & Deutsch \\
\hline Selbstidentifikation in der ersten Person & 1 & 7 & 1 \\
\hline Identifikation "aus dem Off” & 4 & 6 & 6 \\
\hline Identifikation über das Produkt & 0 & 0 & 1 \\
\hline gesamt & 5 & 13 & 8 \\
\hline
\end{tabular}

Tabelle 3: Identifikation des Senders im Werbetext 
Tabelle 3 zeigt, dass die Selbstidentifikation des Senders im spanischen Korpus am häufigsten belegt ist. Wenn der Anbieter sich selbst vorstellt, wird die Kommunikation persönlicher, sodass die Selbstidentifikation als indirekter Adressierungsmarker zu betrachten ist.

Ein weiterer Adressierungsmarker ist die Form, in der sich der Sender an den Adressaten wendet. Diese Form hängt normalerweise davon ab, welche Intention der Sender mit seiner Äußerung verfolgt. In Werbetexten können wir davon ausgehen, dass die wichtigste Intention darin besteht, den Adressaten zum Kauf des angebotenen Produkts zu bewegen. Es handelt sich daher eindeutig um eine appellative Intention (vgl. Nord 1997:42f.). Die appellative Intention kann direkt oder indirekt ausgedrückt werden. Ein indirekter Appell wird über die Darstellung der (positiven) Eigenschaften des Produkts realisiert (dabei wird die referentielle Funktion als Mittel zum Appell genutzt) oder über die Kundgabe eines (positiven) Werturteils (d.h. über die expressive Funktion, vgl. Nord 1997:43). Im Zusammenhang mit der Adressierung interessiert uns hier jedoch vor allem die direkte Verbalisierung der appellativen Funktion in Form von Vorschlägen, Ratschlägen, Einladungen usw. Daher werden alle die Formen registriert, die sich auf den Empfänger beziehen, also:

- Imperative (3.2-13, 3.2-14, 3.2-15) und imperativische oder anleitende Infinitive (3.2-16, 3.2-17), die bereits als solche eine Adressierung enthalten;

- alle Referenzen auf die zweite Person in der vertraulichen oder der Höflichkeitsform (durch das Personal- oder ein Possessivpronomen, im Spanischen auch nur in der Verb-Endung), welche die folgenden Sprechakte markieren: Vorschlag (3.2-18, 3.2-19, auch 3.121), Anleitung (3.2-19, 3.2-20), Empfehlung (3.2-22, 3.2-23, 3.224), Ausdruck der Möglichkeit (3.2-25), Versprechen (3.2-26, 3.227, 3.2-28), Hinweis (3.2-29, 3.2-30), sowie Ausrufe (3.2-31), Warnungen (3.2-32), Lob (3.2-33), Tadel (3.2-34), Begrüßung, Verabschiedung $(3.1-5,3.1-27)$ und Hinweis auf eine Notwendigkeit (3.2-35), sofern sie nicht so selten belegt sind, dass wir sie unter "Andere Formen" zusammenfassen;

- an den Adressaten gerichtete direkte oder rhetorische Fragen (siehe 3.1-6 bis 3.1-11);

- Referenzen auf die Zielgruppe in der dritten Person (3.1-15, 3.225, 3.2-36); und

- unpersönlich formulierte Verallgemeinerungen (z.B. es. hay que, reflexives Passiv, 3.2-23, unpersönliches Pronomen, z.B. de. man), bei denen der Adressat eingeschlossen ist (3.2-37, 3.2-38, 3.2-39), sowie die bereits erwähnten Slogans in Sprichwortform im Deutschen (3.2-40). 


\begin{tabular}{|c|c|}
\hline $3.2-13$ & $\begin{array}{l}\text { Treat your taste buds to the unexpected delights of more than } \\
100 \text { domestic grape varieties. (ALK) }\end{array}$ \\
\hline $3.2-14$ & Escápate a la aventura con Viajes El Corte Inglés. (TOU) \\
\hline $3.2-15$ & Entdecken Sie neue faszinierende Seiten der Literatur. (INF) \\
\hline $3.2-16$ & $\begin{array}{l}\text { Compartir. CHROME AZZARO (KOS, das Bild zeigt Groß- } \\
\text { vater, Vater und Sohn) }\end{array}$ \\
\hline $3.2-17$ & Jetzt Probe fahren. (AUT) \\
\hline $3.2-18$ & $\begin{array}{l}\text { No pasa nada porque te reserves un poco de tiempo para ti } \\
\text { mismo. (TEL) }\end{array}$ \\
\hline $3.2-19$ & Unser Geschenktipp: schnucklige Eierwärmer. (MOD) \\
\hline $3.2-20$ & $\begin{array}{l}\text { Cómo llamar de Barclona a Vigo ahorrándote hasta un } 25 \% . \\
\text { (TEL) }\end{array}$ \\
\hline $3.2-21$ & Wie man Mitarbeiter motiviert: Schmeißen Sie alle raus! (FIN) \\
\hline $3.2-22$ & Your car should have a lot of space in it, not your wallet. (AUT) \\
\hline $3.2-23$ & $\begin{array}{l}\text { "La vida debe vivirse hacia adelante, pero sólo se comprende } \\
\text { hacia atrás." Sören Kierkegaard. (ALK) }\end{array}$ \\
\hline $3.2-24$ & $\begin{array}{l}\text { Außerdem sollten Sie ein Auge auf die Leistungswerte werfen. } \\
\text { (BÜR) }\end{array}$ \\
\hline $3.2-25$ & $\begin{array}{l}\text { Damit können alle 190er-Fahrer ab sofort rechnen. Das Spar- } \\
\text { buch für den 190er. (AUT) }\end{array}$ \\
\hline $3.2-26$ & $\begin{array}{l}\text { There's a storm brewing... and at its eye you'll find the new } \\
\text { Puma Thunder. (AUT) }\end{array}$ \\
\hline $3.2-27$ & Juntos hacemos tu vida mejor. (FOT) \\
\hline $3.2-28$ & $\begin{array}{l}\text { Wie auch immer in Zukunft informiert wird: Sie hören von uns. } \\
\text { (INF) }\end{array}$ \\
\hline $3.2-29$ & $\begin{array}{l}\text { Halfprice golf irons. Here's an ideal opportunity for anyone buy- } \\
\text { ing their first full set of irons... (GES) }\end{array}$ \\
\hline $3.2-30$ & Hier geht's rein. (BÜR, mit Bezug auf das Internet) \\
\hline $3.2-31$ & ¡La cuenta atrás ha comenzado! (KOM) \\
\hline $3.2-32$ & Safety first: $\underline{w w w . d r e s d n e r-b a n k . d e}(\mathrm{FIN})$ \\
\hline $3.2-33$ & Sabes eligir. (ALK) \\
\hline $3.2-34$ & $\begin{array}{l}\text { Acabas de perder una oportunidad de oro. ... Búscalos en el inte- } \\
\text { rior de los sobrecitos de Nescafé. Y no dejes pasar ni una sola } \\
\text { oportunidad de encontrar oro. (NAH) }\end{array}$ \\
\hline $3.2-35$ & $\begin{array}{l}\text { Auf dem Weg zum Parkplatz brauchen Sie zwei Airbags. } \\
\text { (MOD) }\end{array}$ \\
\hline $3.2-36$ & $\begin{array}{l}\text { Gucci rush, for men. The new fragrance for men from Gucci } \\
\text { (KOS, spanisch) }\end{array}$ \\
\hline
\end{tabular}




\begin{tabular}{|l|l|}
\hline 3.2-37 & $\begin{array}{l}\text {...everyone's favourite little black number (ALK, Freixenet black } \\
\text { lable) }\end{array}$ \\
\hline $3.2-38$ & $\begin{array}{l}\text { 2000 - ¿principio o fin? Hay fechas que marcan eras. El año } \\
2.000 \text { será una de ellas. Pero no todos están preparados para las } \\
\text { consecuencias que se derivarán de la entrada en el nuevo mile- } \\
\text { nio; por eso para algunos el 2.000 será el principio y para otros } \\
\text { el final. (BÜR) }\end{array}$ \\
\hline $3.2-39$ & $\begin{array}{l}\text { Good taste has many faces. ... Jeder informierte Erwachsene } \\
\text { sollte die Freiheit haben, sich für den Genuss von Tabak zu ent- } \\
\text { scheiden. (TAB) }\end{array}$ \\
\hline $3.2-40$ & $\begin{array}{l}\text { Wer hoch hinaus will, kann sich keine Abstürze leisten. Sichern } \\
\text { Sie sich darum einfach mit einem Server von MAXDATA ab. } \\
\text { (BÜR) }\end{array}$ \\
\hline
\end{tabular}

Werbetexte ohne jegliche Adressierung sind vergleichsweise selten (18 im englischen, 15 im spanischen und 19 im deutschen Korpus). In vielen Werbetexten sind mehrere der genannten Addressierungsmarker belegt. Tabelle 4 verdeutlicht ihre Verteilung im Korpus.

\begin{tabular}{|l|r|r|r|r|r|r|}
\hline $\begin{array}{l}\text { Appellative Adressierungs- } \\
\text { formen }\end{array}$ & \multicolumn{2}{|c|}{ Englisch } & \multicolumn{2}{l|}{ Spanisch } & \multicolumn{2}{l|}{ Deutsch } \\
\hline Imperativ & 64 & $55,7 \%$ & 71 & $58,7 \%$ & 38 & $25,5 \%$ \\
\hline Infinitiv & 0 & $0,0 \%$ & 1 & $0,8 \%$ & 14 & $9,4 \%$ \\
\hline Vorschlag & 0 & $0,0 \%$ & 2 & $1,6 \%$ & 1 & $0,7 \%$ \\
\hline Anleitung & 0 & $0,0 \%$ & 1 & $0,8 \%$ & 1 & $0,7 \%$ \\
\hline Empfehlung & 1 & $0,8 \%$ & 1 & $0,8 \%$ & 2 & $1,3 \%$ \\
\hline Möglichkeit & 2 & $1,7 \%$ & 0 & $0,0 \%$ & 5 & $3,4 \%$ \\
\hline Versprechen & 18 & $15,7 \%$ & 18 & $14,9 \%$ & 11 & $7,3 \%$ \\
\hline Frage & 11 & $9,6 \%$ & 12 & $9,9 \%$ & 11 & $7,3 \%$ \\
\hline Hinweis & 2 & $1,7 \%$ & 0 & $0,0 \%$ & 1 & $0,7 \%$ \\
\hline Referenz auf die Zielgruppe & 4 & $3,5 \%$ & 3 & $2,5 \%$ & 5 & $3,4 \%$ \\
\hline Verallgemeinerung & 11 & $9,6 \%$ & 6 & $5,0 \%$ & 53 & $35,6 \%$ \\
\hline Andere Formen & 2 & $1,7 \%$ & 6 & $5,0 \%$ & 7 & $4,7 \%$ \\
\hline Gesamt & 115 & $100 \%$ & 121 & $100 \%$ & 149 & $100 \%$ \\
\hline
\end{tabular}

Tabelle 4: Appellative Adressierung in Werbetexten 
Die hohe Zahl von Adressierungsformen im deutschen Korpus erscheint auf den ersten Blick überraschend. Mehr als die Hälfte von ihnen (50,1\%) entfällt jedoch auf die Gruppe der neutralen Formen (Infinitive, Anleitungen, Hinweise, Zielgruppenreferenz und Verallgemeinerung), die in den beiden anderen Teilkorpora eine erheblich geringere Rolle spielen (13,1\% im englischen und 8,3\% im spanischen Korpus). Dadurch ist die Appellativität wesentlich "versteckter".

\subsection{Beziehungsindikatoren in Werbetexten}

Der hohe Anteil neutraler Strukturen im deutschen Korpus hat auch Konsequenzen für die Markierung der sozialen Beziehung zwischen den Kommunikationspartnern. Wir hatten ja bereits festgestellt, dass diese Beziehung zunächst vor allem durch die verwendeten Anredeformen markiert wird. In einer normalen Kommunikationssituation erwarten wir, dass eine Person, die man nicht kennt, in der Höflichkeitsform angesprochen wird, sofern es sich nicht um eine asymmetrische Relation wie zwischen Erwachsenen und Kindern oder höher- und niedriger gestellten Personen handelt. In den Werbetexten ist das anders. Ausgenommen in den englischen Texten, wo ja nur eine Anredeform zur Verfügung steht, finden wir in Werbetexten beide Anredeformen. Dabei ist in den deutschen Werbetexten jedoch die vertrauliche Anrede mit $d u$ sehr selten und auf ganz bestimmte, für eine jugendliche Zielgruppe gedachte Produkte beschränkt. Tabelle 5 zeigt die Verteilung der Anredeformen, wobei die englischsprachigen Imperative und die imperativischen Infinitive im deutschen und spanischen Korpus als "neutrale" Formen der Anrede betrachtet werden. Die Prozentzahlen beziehen sich auf den Anteil der betreffenden Form an den Werbungen mit Anrede; bei den englischen Texten werden auch Imperative als explizite Anrede betrachtet, obwohl kein Pronomen verwendet wird.

\begin{tabular}{|c|c|c|c|c|c|c|}
\hline Anredeformen & \multicolumn{2}{|c|}{ Englisch } & \multicolumn{2}{|c|}{ Spanisch } & \multicolumn{2}{|c|}{ Deutsch } \\
\hline formelle Anrede (usted/es, Sie) & 71 & $100 \%$ & 28 & $40,0 \%$ & 46 & $77,9 \%$ \\
\hline vertrauliche Anrede (tú, $d u)$ & & & 40 & $57,1 \%$ & 2 & $3,4 \%$ \\
\hline neutrale Anrede & 0 & $0,0 \%$ & 2 & $2,9 \%$ & 11 & $18,7 \%$ \\
\hline Werbetexte mit Anrede & 71 & $100 \%$ & 70 & $100 \%$ & 59 & $100 \%$ \\
\hline
\end{tabular}

Tabelle 5: Anredeformen in Werbetexten

Wenn man von den Anredeformen ausgeht, scheinen die spanischen Werbetexte eine wesentlich vertraulichere Beziehung zu signalisieren als die deutschen, die offenbar eine größere soziale Distanz markieren. Das könnte den Schluss nahelegen, dass die spanischen Textproduzenten die Empfänger von Gleich zu Gleich behandeln, als ob sie sie schon lange kennen würden, 
während die deutschen Textproduzenten eine formellere Beziehung bevorzugen - beides würde auch dem Gebrauch der Anredeformen zwischen Unbekannten im "richtigen Leben" wiederspiegeln. Aber wir haben ja bereits darauf hingewiesen, dass neben der Anrede auch das gewählte Register eine wichtige Rolle für die Definition der Beziehung spielt. Daher könnte das Register in Kulturen mit weniger differenzierten Anredeformen eine wichtigere Rolle spielen als dort, wo eine Differenzierung über das Anredepronomen möglich ist.

Unter "Register" verstehen wir mit Crystal \& Davy (1969) die Gesamtheit der lexikalischen, stilistischen, rhetorischen und gegebenenfalls phonologischen Mittel, die die Sprachverwendung als zu einer bestimmten kommunikativen Situation zugehörig markieren. Im Zusammenhang mit dem Beziehungsaspekt der phatischen Funktion beschränken wir uns hier auf die Mittel, die den Unterschied zwischen formeller und informeller Sprachverwendung kennzeichnen. Wir unterscheiden dabei syntaktische und lexikalische Registermarkierungen.

Im englischen Korpus sind folgende Markierungen eines informellen Registers zu verzeichnen: Im Bereich der Syntax finden wir Ellipsen (3.3-1, siehe auch 3.1-11, 3.1-15), Parenthesen (3.1-16), Satzabbrüche (3.1-19, 3.2-27), Relativsätze ohne Pronomen (3.3-2), Satzendstellung der Präposition (3.3-3). Im Bereich der Lexik sind zahlreiche Wörter und Ausdrücke zu verzeichnen die im Dictionary of Contemporary English als informal oder not formal markiert sind (z.B. to get the shivers, get ready, Xmas, telly, posh, ultimate) oder sogar als slang (z.B. to twig im Sinne von 'kapieren', to give an edge, whatever turns you on, what the hell, 3.3-4), Modalpartikeln (besonders just, 3.3-5), Kontraktionen mit Apostroph, wie sie in der gesprochenen Sprache vorkommen (z.B. doesn't, don't, isn't, I'll, I've, we'll, here's, 3.3-6), sowie einige Interjektionen (3.1-17).

\begin{tabular}{|c|c|}
\hline 3.3-1 & $\begin{array}{l}\text { Nothing on TV. No new message. Off-licence shut. What the } \\
\text { hell. - Everything to colour and care for your hair. (KOS) }\end{array}$ \\
\hline $3.3-2$ & $\begin{array}{l}\text { Isn't it nice to get something you really want for Christmas for a } \\
\text { change? (TEL) }\end{array}$ \\
\hline $3.3-3$ & $\begin{array}{l}\text { Men, difficult to buy for? (MOD) / Love the skin you're in. } \\
\text { (GES) }\end{array}$ \\
\hline $3.3-4$ & $\begin{array}{l}\text { Business travel doesn't have to B A rip-off. (TRA, British } \\
\text { Airways) }\end{array}$ \\
\hline $3.3-5$ & $\begin{array}{l}\text { So I just drive and it gives me the right directions? Yes! Just lis- } \\
\text { ten to the clear voice directions on this satellite navigator, exclu- } \\
\text { sive to Dixons. (TRA) }\end{array}$ \\
\hline $3.3-6$ & $\begin{array}{l}\text { You ain't seen nothing 'til you've seen our gift guide. If you think } \\
\text { you've seen every Christmas gift call for our freee Ultimate Gift } \\
\text { Guide today. (MOD) }\end{array}$ \\
\hline
\end{tabular}


Im spanischen Korpus wird das informelle Register durch folgende Markierungen signalisiert: in der Syntax Ellipsen (3.3-7, siehe auch 3.1-10, 3.1-22), Parenthesen (3.3-8), Satzabbrüche (3.1-4), Fokusstrukturen (3.3-9); in der Lexik Wörter und Ausdrücke, die laut Diccionario del español actual als coloquial oder popular konnotiert sind (3.3-10), ein Diskursmarker (3.1-3) und eine Diminutivform (3.2-34).

\begin{tabular}{|l|l|}
\hline $3.3-7$ & $\begin{array}{l}\text { La prensa está de acuerdo: un turismo que parece un deportivo. } \\
\text { O al revés. (AUT) }\end{array}$ \\
\hline $3.3-8$ & $\begin{array}{l}\text { La única cosa que no cuestionamos (al menos de momento). } \\
\text { (AUT) }\end{array}$ \\
\hline $3.3-9$ & $\begin{array}{l}\text { Invierte sabiendo que en cualquier momento puedes recuperar tu } \\
\text { dinero. El camino para realizar tos sueños sólo lo marcas tú. } \\
\text { (FIN) }\end{array}$ \\
\hline $3.3-10$ & Baje de las nubes y toque de pies en Ofiprix. (BÜR) \\
\hline
\end{tabular}

Das deutsche Korpus weist folgende Markierungen eines informellen Registers auf: In der Syntax ebenfalls Ellipsen (3.3-11, auch 3.1-25) und Satzabbrüche, in der Lexik Wörter und Ausdrücke, die im DudenWörterbuch der deutschen Sprache als umgangssprachlich (z.B. Job, loslegen: 3.1-8, Geld machen, mal anstatt einmal, 3.3-12) oder salopp (z.B. einloggen) konnotiert sind, sprechsprachliche Kontraktionen (etwa hat's: 3.3-13, geht's: 3.2-30), Modalpartikeln und Diskursmarker (z.B. ja, doch, einfach: 3.3-12, 3.2-40, na: 3.3-13) und ein anaphorisch gebrauchter Artikel.

\begin{tabular}{|l|l|}
\hline 3.3-11 & $\begin{array}{l}\text { Mausi tot. Gerade noch Herzensbrecher und plötzlich aus die } \\
\text { Maus. (GES) }\end{array}$ \\
\hline 3.3-12 & $\begin{array}{l}\text { Wer an der Börse Geld machen will, braucht gute Nerven. } \\
\text { Fragen Sie doch einfach mal. (FIN) }\end{array}$ \\
\hline 3.3-13 & $\begin{array}{l}\text { Der ideale Platz zum Surfen. debitel gibt Ihnen die Freiheit, sich } \\
\text { auch mal abseits der üblichen Pfade ins Internet einzuloggen. } \\
\text { debitel: } \text { na, hat's geklingelt? (TEL) }\end{array}$ \\
\hline
\end{tabular}

Tabelle 6 zeigt die Häufigkeit der informellen Registermarkierungen in den Korpustexten. Schon die absolute Zahl der Markierungen ist in den drei Teilkorpora sehr verschieden: In den englischen Texten sind sie am häufigsten und in den spanischen Texten am seltensten belegt, was der oben erwähnten Verwendung der Anredeformen entspricht. Wenn die geringe soziale Distanz bereits durch die informelle Anrede markiert ist, sind Registermarker nicht mehr so notwendig, um die gewünschte Vertraulichkeit zu suggerieren. 


\begin{tabular}{|l|r|r|r|r|r|r|}
\hline Marker & \multicolumn{2}{|c|}{ Englisch } & \multicolumn{2}{c|}{ Spanisch } & \multicolumn{2}{|c|}{ Deutsch } \\
\hline Ellipse & 25 & $25,8 \%$ & 7 & $36,7 \%$ & 22 & $36,1 \%$ \\
\hline Parenthese & 3 & $3,1 \%$ & 1 & $5,3 \%$ & 0 & $0,0 \%$ \\
\hline Satzabbruch (Anakoluth) & 3 & $3,1 \%$ & 4 & $21,0 \%$ & 5 & $8,2 \%$ \\
\hline andere syntaktische Marker & 5 & $5,2 \%$ & 1 & $5,3 \%$ & 0 & $0,0 \%$ \\
\hline $\begin{array}{l}\text { als umgangssprachlich } \\
\text { markierte Lexik }\end{array}$ & 14 & $14,4 \%$ & 2 & $10,5 \%$ & 17 & $27,9 \%$ \\
\hline als salopp markierte Lexik & 8 & $8,2 \%$ & 1 & $5,3 \%$ & 2 & $3,3 \%$ \\
\hline sprechsprachliche Formen & 31 & $31,9 \%$ & 0 & $0,0 \%$ & 3 & $4,9 \%$ \\
\hline Partikeln und Diskursmarker & 5 & $5,2 \%$ & 1 & $5,3 \%$ & 10 & $16,4 \%$ \\
\hline Interjektionen & 3 & $3,1 \%$ & 1 & $5,3 \%$ & 1 & $1,6 \%$ \\
\hline andere lexikalische Marker & 0 & $0,0 \%$ & 1 & $5,3 \%$ & 1 & $1,6 \%$ \\
\hline gesamt & 97 & $100 \%$ & 19 & $100 \%$ & 61 & $100 \%$ \\
\hline
\end{tabular}

Tabelle 6: Markierung eines informellen Registers

Die Analyseergebnisse sind jedoch nicht nur quantitativ, sondern auch qualitativ interessant. Im spanischen Korpus stellen die syntaktischen Marker mehr als 68\%, im englischen Korpus nur 37\% und im deutschen Korpus 44\%. Dafür sind in den letzteren die lexikalischen Marker nicht nur häufiger, sondern auch vielfältiger. Das liegt gewiss zum Teil an der Tatsache, dass im Englischen und Deutschen die Stilebenen ober- bzw. unterhalb des mittleren, neutralen Niveaus schärfer von einander abgegrenzt sind als im Spanischen (vgl. beispielsweise die fünf "Formalitätsstufen" bei Crystal \& Davy 1969: 74 , die von frozen, formal, consultative bis casual und intimate reichen). Die unterschiedliche Frequenz und Distribution der Marker muss jedoch auf kulturelle Konventionen zurückgeführt werden. Auffällig ist der Anteil der sprechsprachlichen Formen im Englischen!

\begin{tabular}{|l|l|}
\hline 3.3-14 & $\begin{array}{l}\text { It takes more than } 8 \text { years for a child's immune system to fully } \\
\text { develop. Until then nothing reduces the symptoms of fever fas- } \\
\text { ter or for longer than Nurofen for Children. NUROFEN } \\
\text { KNOWS CHILDREN. (GES) }\end{array}$ \\
\hline $3.3-15$ & $\begin{array}{l}\text { Thermal S. With the benefits of Vichy thermal spa water to inten- } \\
\text { sively rehydrate your skin. (GES) }\end{array}$ \\
\hline $3.3-16$ & $\begin{array}{l}\text { LOEWE obsequia a los compradores de cualquiera de los } \\
\text { siguientes modelos... (INF) }\end{array}$ \\
\hline
\end{tabular}


3.3-17 Refinando la perfección. El tweeter con la tecnología Nautilus, que ha hecho merecedores de los más encendidos elogios a los modelos de la Serie Nautilus 800, ha sido ahora incorporada [sic] a la Serie 600 S2. (HAU)

Abschließend ist noch festzustellen, dass nur in ganz wenigen Werbetexten des Korpus ein formelles oder gehobenes Register markiert wird. Die Beispiele 3.3-14 und 3.3-15 repräsentieren ein consultative register (nach Christal \& Davy 1969) und in Beispiel 3.1-19 erkennt man einen leicht formelleren Ton, weil es sich offensichtlich an ein gebildetes (und vermutlich wohlhabenderes) Publikum richtet, das die Anspielung erkennt. Die Beispiele 3.3-16 und 3.3-17, bei denen es sich um Übersetzungen handeln könnte, klingen fast parodistisch, zumindest Beispiel 3.3-17, bei dem der sehr gehobene Stil der Situation und der Textsorte unangemessen und daher überzogen erscheint. Im deutschen Korpus sind ein paar wenige Texte belegt, die stilistisch unmarkiert sind, jedoch kein einziger mit einem gehobenen oder literarischen Stil.

\section{Schlussbemerkungen}

Abschließend betrachten wir die Analyseergebnisse im Überblick. Tabelle 7 macht deutlich, dass die Unterschiede zwischen den drei betrachteten Kulturen bei den meisten Indikatoren, sogar bei der direkten Anrede und im Formalitätsgrad, eher graduell sind. Die Kontakteröffnungs- und Selbstidentifikationsindikatoren, die für eine Kommunikation zwischen Unbekannten sonst typisch sind, spielen hier eine eher marginale Rolle. Das lässt darauf schließen, dass es den Textproduzenten eher darum geht, bei den Adressaten den Eindruck zu erwecken, dass sie mit einem alten Bekannten sprechen und nicht mit einer Person, die sie nie zuvor gesehen haben. Die Kontakterhaltsindikatoren sind dagegen relativ häufig; dass sie im spanischen Korpus weniger oft belegt sind als in den beiden anderen, kann Zufall und auf den geringen Umfang der Korpora zurückzuführen sein. In allen drei Teilkorpora stellen die appellativen Sprechakte den größten Anteil bei den phatischen Indikatoren. Das erklärt sich aus der vorrangigen Funktion von Werbetexten allgemein, die Zielgruppe zum Erwerb des angebotenenen Produkts zu "überreden". Hier stellen wir fest, dass im englischen und spanischen Korpus die Verwendung persönlicher Appellformen überwiegt, während im deutschen Korpus unpersönliche oder indirekte Appellformen bevorzugt werden. 


\begin{tabular}{|l|r|r|r|r|r|r|}
\hline Phatische Funktionsindikatoren & \multicolumn{2}{|c|}{ Englisch } & \multicolumn{2}{|c|}{ Spanisch } & \multicolumn{2}{c|}{ Deutsch } \\
\hline Kontakteröffnung & 15 & $4,1 \%$ & 13 & $4,8 \%$ & 10 & $2,9 \%$ \\
\hline Senderidentifikation & 5 & $1,4 \%$ & 13 & $4,8 \%$ & 8 & $2,3 \%$ \\
\hline Kontakterhalt & 60 & $16,5 \%$ & 35 & $12,9 \%$ & 55 & $16,1 \%$ \\
\hline Appellative Formen & 115 & $31,7 \%$ & 121 & $44,6 \%$ & 149 & $43,6 \%$ \\
\hline Direkte Anrede & 71 & $19,6 \%$ & 70 & $25,8 \%$ & 59 & $17,3 \%$ \\
\hline Informelles Register & 97 & $26,7 \%$ & 19 & $7,1 \%$ & 61 & $17,8 \%$ \\
\hline Indikatoren gesamt & 363 & $100 \%$ & 271 & $100 \%$ & 342 & $100 \%$ \\
\hline
\end{tabular}

Tabelle 7: Phatische Funktionsindikatoren in Werbetexten

Am größten sind die Unterschiede zwischen den drei Teilkorpora im Hinblick auf die Form der Beziehungsindikatoren, das heißt, bei der Anrede und der Markierung eines informellen Registers.

Der Gebrauch der pronominalen Anredeformen entspricht in allen drei Teilkorpora dem üblichen Gebrauch in anderen entsprechenden Situationen der betreffenden Kultur. In Deutschland ist die obligatorische Anrede zwischen Erwachsenen (ab ca. 16 Jahren), die sich nicht kennen, das Sie; eine Ausnahme stellt nur die allgemeine Bevorzugung des Duzens zwischen Jugendlichen des gleichen Alters (zumindest außerhalb der Arbeitswelt) und Studierenden an Hochschulen dar. In Spanien ist das Duzen wesentlich weiter verbreitet. Alonso-Cortés (1999:4041) zufolge duzen sich generell Kolleginnen und Kollegen, Gleichaltrige, Lehrende und Lernende an der Universität, geduzt werden aber auch Verkäufer/innen, Taxifahrer/innen, Kellner/innen, besonders wenn sie jünger sind als der Sprecher, wobei in den letzteren Fällen oft eine asymmetrische Anrede festzustellen ist (vgl. auch Matte Bon 1995:I, 244), die in Deutschland nur in ganz speziellen Situationen vorkommt (zum Beispiel zwischen den Eltern erwachsener Kinder und deren Freunden, besonders wenn man sie schon von Klein auf kennt). Eine kleine Analyse von Stellenanzeigen (vgl. Nord 2003:18ff.) zeigt, dass bei der Aufforderung, sich zu bewerben, sämtliche Texte mit einer direkten Anrede im Deutschen die Sie-Form (auch bei Anzeigen, die sich explizit an junge Schulabgänger/innen wenden) und im Spanischen die DuForm verwenden. Im Spanischen ist also in Erstkontaktsituationen durchaus eine Differenzierung der Anrede festzustellen, während im Deutschen in diesen Situationen das Siezen im Prinzip die einzige mögliche Form der Anrede ist, will man sich nicht den Vorwurf "plumper Vertraulichkeit" zuziehen.

Wir hatten oben gesagt, dass dort, wo keine alternative Formulierung zur Verfügung steht, die verwendete Form keinen stilistischen Wert hat, dass aber die Entscheidung darüber, ob man eine Funktion überhaupt versprachlicht, mit nichtsprachlichen Mitteln oder gar nicht ausdrückt, sehr wohl kulturellen Konventionen unterliegt. Ob also die Werbetexte eine Anrede ent- 
halten, ist kulturspezifisch - welche Anrede gewählt wird, ist nur dann konventionsbedingt, wenn mehr als eine Anredeform zur Verfügung steht. In allen drei hier untersuchten Sprachen gibt es neben der Möglichkeit der Nicht-Anrede verschiedene Formen der Anrede: die "unpersönliche" und die "persönliche" Anrede, wobei letztere vom Sprachsystem her im Deutschen und Spanischen, in der Sprachwirklichkeit zwischen unbekannten Personen jedoch nur im Spanischen nach "vertraulich" und "distanziert" unterschiedlich ist.

Um eine deutlich als vertraulich oder solidarisch gekennzeichnete Beziehung zu markieren und den Adressaten durch An-Reden zu ÜberReden, müssen daher die Verfasser von Werbetexten in England und Deutschland auf Registermarkierungen zurückgreifen. Anredeformen und Registermarkierungen wirken also bei der Schaffung einer vertraulichen sozialen Beziehung komplementär. Im Englischen, wo eine Differenzierung der Anredepronomina fehlt, wird die vertrauliche Atmosphäre durch einen informellen, zuweilen geradezu saloppen Sprachstil mit zahlreichen umgangs- und vor allem sprechsprachlichen Merkmalen geschaffen. Das Gleiche gilt für das Deutsche, wo die Modalpartikeln, ebenfalls ein Merkmal gesprochener, informeller Sprache, eine wichtige Rolle spielen. Im Spanischen dagegen werden die vertrauliche Anrede oder eine Kombination aus vertraulicher Anrede und informellem Register bevorzugt, um eine informelle Beziehung herzustellen. Wegen der fließenden Grenzen zwischen den Stilebenen im Bereich der Lexik werden dabei syntaktische Marker bevorzugt, wie sie für die gesprochene Sprache charakteristisch sind. Natürlich sind diese Ergebnisse angesichts des geringen Umfangs des Korpus mit Vorsicht zu genießen und sollten anhand eines umfangreichen, möglichst elektronisch gespeicherten Korpus überprüft werden. Dennoch kann die am Anfang aufgestellte These, dass die phatische Funktion sich im Wesentlichen auf die Konventionalität der verwendeten (hier im Wesentlichen sprachlichen) Mittel stützt, durchaus als gesichert angenommen werden. Die Frage, ob es hier zum Teil nicht doch um sprachliche und nicht um kulturelle Phänomene geht, lässt sich nur durch einen Vergleich des hier benutzten Korpus mit Korpora aus Werbetexten feststellen, die in anderen englisch-, deutsch- und spanischsprachigen Ländern verwendet werden. Dies muss jedoch späteren Untersuchungen vorbehalten bleiben.

\section{Bibliographie}

Alonso-Cortés, Ángel (1999). "Las construcciones exclamativas. La interjección y las expresiones vocativas." Ignacio Bosque Múñoz \& Violeta Demonte Barreto (1999). Gramática descriptiva de la lengua española (GDLE). Madrid: Espasa Calpe. RAE - Colección Nebrija y Bello. Kap. 62, 39944050.

Álvarez Lugrís, Alberto (2001). Estilística Comparada da Traducción. Proposta metodolóxica e aplicación práctica ó estudio do corpus TEXTRA de traduccións do inglés ó galego. Vigo: Servicio de Publicacións de la Universidade de Vigo. 
Crystal, David \& Derek Davy (1969). Investigating English Style. London: Longman.

Diccionario del Español Actual = Seco, Manuel, Andrés, Olimpia \& Gabino Ramos (Hrsg.) (1999). Diccionario del Español Actual. 2 Bde. Madrid: Aguilar.

Dictionary of Contemporary English = Procter, Paul et al. (Hrsg.) (1978). Dictionary of Contemporary English. Longman-Langenscheidt.

Duden-Das große Wörterbuch der deutschen Sprache = Drosdowski,Günther et al. (Hrsg.) (1993). Duden-Das große Wörterbuch der deutschen Sprache. 7 Bde. Mannheim: Dudenverlag.

Cartagena, Nelson \& Hans-Martin Gauger (1989). Kontrastive Grammatik Spanisch-Deutsch. Mannheim: Duden-Verlag.

Jakobson, Roman (1960). "Linguistics and Poetics." Thomas A. Sebeok (Hrsg.) (1960). Style in Language. Cambridge/Mass.: MIT Press, 350-377.

Lewis, David K. (1969). Conventions. A Philosophical Study. Cambridge/Mass.: Harvard University Press.

Matte Bon, Francisco (1995). Gramática Comunicativa del español. 2 Bde. Madrid: edelsa.

Nord, Christiane (1997). Translating as a Purposeful Activity. Functionalist Approaches Explained. Manchester: St. Jerome.

Nord, Christiane (2003). Kommunikativ handeln auf Spanisch und Deutsch. Ein übersetzungsorientierter, korpusgestützter Sprach- und Stilvergleich. Wilhelmsfeld: Gottfried Egert (im Druck).

Packard, Vance (1967). The Hidden Persuaders. 20. Aufl. New York: McKay.

Valdés Rodríguez, M. Cristina (1999). La traducción publicitaria como acto de comunicación intercultural. Diss., Depto. de Filología Anglogermánica y Francesa, Universidad de Oviedo.

Vinay, Jean-Paul \& Jean Darbelnet (1958). Stylistique comparée du français et de l'anglais. Méthode de traduction. Paris: Didier.

\section{Korpus}

Englischsprachige Anzeigenwerbung (Großbritannien)

1) Daily Telegraph, 12/2001

2) Daily Mail, 12/2001

3) The Mail on Sunday, London, 12/2001

4) The Observer, London, 12/2001

5) Life. The Observer Magazine, London, 12/ 2001

6) Newwoman, London, 01/2002

Deutschsprachige Anzeigenwerbung (Deutschland)

1) Nord, Oliver (1999). Über die Funktionen von Wortspielen in Werbeanzeigen. Untersuchungen zur Werbewirksamkeit von Wortspielen anhand eines Zeitschriftenkorpus. Unveröfftl. Magisterarbeit. Universität Heidelberg.

2) Die Marke, Beilage der überregionalen Tageszeitung Süddeutsche Zeitung, aus Anlass des Deutschen Marketingtags 2001.

3) DB mobil, Kundenzeitschrift der Deutschen Bahn AG, 12/2001.

4) Der Spiegel, Nachrichtenmagazin, 05/2001

5) Lufthansa Magazin, 11/2001, 12/2001, 01/2002

Spanischsprachige Anzeigenwerbung (Spanien)

1) Actualidad Económica, Madrid 5-11 März 2001.

2) The Tourist, monatlich erscheinende Informationsbroschüre für Touristen, die in spanischen Hotels ausliegt, 2/01. 
3) Cosmopolitan España 7/96.

4) Ronda Iberia, Inflight-Magazin der spanischen Fluglinie Iberia, 3/98, 5/98.

5) El País Semanal, Wochenendbeilage der spanischen Tageszeitung El País, 10/96, 10/97, 10/98

6) Inversión. Semanario de ahorro y gestión de patrimonios, spanisches Wirtschaftsmagazin, 23/02-01/03, 2001

7) Capital, spanisches Wirtschaftsmagazin, 6/01

8) El País, in Madrid erscheinende überregionale Tageszeitung, 11/01

${ }^{1}$ Die Produktkategorien werden in Form von Siglen angegeben: FIN = Banken, Versicherungen, Investmentfirmen, ALK = Alkoholische Getränke, TEL = Telekommunikation, TRA $=$ Transport und Verkehr, HAU $=$ Haushalt, $\mathrm{KOM}=$ Handel, AUT $=$ Autos und Zubehör, Vermietung, KOS $=$ Kosmetik, NAH = Nahrungsmittel, $\mathrm{TOU}=$ Tourismus, $\mathrm{TAB}=$ Tabakwaren, $\mathrm{INF}=$ Informationsmedien, Bücher, MOD = Mode, BIL = Bildung und Ausbildung, BÜR = Büroausstattung, GES = Gesundheit.

${ }^{2}$ Wir verwenden zur Spezifizierung der Sprachen die Abkürzungen der DIN-Norm: en. $=$ Englisch, es. $=$ Spanisch, de. $=$ Deutsch.

${ }^{3}$ Es gibt allerdings bestimmte Anzeigenserien, in denen ab der zweiten Anzeige bereits eine gewisse Vertrautheit zwischen Sender und Empfänger vorausgesetzt wird. Sie sind im Korpus jedoch nicht belegt. 